\title{
Fine Structure of Parvocellular Receptive Fields in the Primate Fovea Revealed by Laser Interferometry
}

\author{
Matthew J. McMahon, Martin J. M. Lankheet, Peter Lennie, and David R. Williams \\ Center for Visual Science, University of Rochester, Rochester, New York 14627
}

Optical blurring in the eye prevents conventional physiological techniques from revealing the fine structure of the small parvocellular receptive fields in the primate fovea in vivo. We explored the organization of receptive fields in macaque parvocellular lateral geniculate nucleus cells by using sinusoidal interference fringes formed directly on the retina to measure spatial frequency tuning at different orientations. Most parvocellular cells in and near the fovea respond reliably to spatial frequencies up to and beyond 100 cycles $/{ }^{\circ}$ of visual angle, implying center input arising mainly from a single cone. Temporal frequency and contrast response characteristics were also measured at spatial frequencies up to 130 cycles $/{ }^{\circ}$. We compared our spatial frequency data with the frequency responses of model recep-

The convergence of signals from individual cone photoreceptors onto subsequent neurons is thought to produce a fundamental limit on contrast sensitivity at high spatial frequencies. Polyak (1941) concluded from anatomical evidence that if individual foveal cones could be stimulated, their impulses "could be transmitted to the brain as single, isolated, independent processes" by the midget ganglion cell system. The spatial density of cones and bipolar cells in the macaque retina (Wässle et al., 1994) suggests that each cone photoreceptor in the fovea and midperipheral retina could provide the input for two midget bipolar cells (one on-center and one off-center), and reconstructions of macaque foveal retinas have shown that there are more than three ganglion cells per foveal cone (Wässle et al., 1989). Anatomical analysis of serial section electron micrographs has shown that primate foveal bipolar cell axon terminals contact one midget ganglion cell exclusively (Calkins et al., 1994), and the same result has been reported for human parafoveal bipolar cells (Kolb and Dekorver, 1991). This anatomical evidence strongly supports the hypothesis that midget ganglion cells maintain the spatial resolution afforded by single cones. However, these "private line" connections might contain crosstalk produced by electrical coupling of neighboring

\footnotetext{
Received June 25, 1999; revised Dec. 21, 1999; accepted Dec. 21, 1999.

This work was supported by a National Science Foundation Graduate Research Fellowship to M.J.M. and National Institutes of Health Grants EY04440, EY04367, EY01319, and EY01711. We thank David Calkins, Harvey Smallman, and Don MacLeod for comments on a draft of this manuscript.

Correspondence should be addressed to Dr. McMahon, Department of Biological Structure, University of Washington, Seattle, WA 98195-7420. E-mail: mm@mattmcmahon.com.

Dr. McMahon's present address: Psychology Department, University of California, San Diego, La Jolla, CA 92093

Dr. Lankheet's present address: H.R. Kruytgebouw, Padualaan 8, $3584 \mathrm{CH}$ Utrecht, The Netherlands.

Dr. Lennie's present address: Center for Neural Science, New York University, New York, NY 10011.

Copyright (C) 2000 Society for Neuroscience $\quad 0270-6474 / 00 / 202043-11 \$ 15.00 / 0$
}

tive fields that estimate the number, configuration, and weights of cones that feed the center and surround. On the basis of these comparisons, we infer possible underlying circuits. Most cells had irregular spatial frequency-response curves that imply center input from more than one cone. The measured responses are consistent with a single cone center together with weak input from nearby cones. By exposing a fine structure that cannot be discerned by conventional techniques, interferometry allows functional measurements of the early neural mechanisms in spatial vision.

Key words: spatial vision; ganglion cells; LGN; parvocellular; acuity; interferometry; retinal circuitry

foveal cones via gap junctions (Cohen, 1965; Raviola and Gilula, 1973; Tsukamoto et al., 1992). There are also two levels of inhibitory lateral connections that could modify the single-cone center signal: horizontal cells (Dacey et al., 1996) and amacrine cells (Calkins and Sterling, 1996). Psychophysical estimates of foveal neural convergence using laser interference fringes have shown that the neural point spread function is slightly larger than a single cone (Campbell and Green, 1965; Sekiguchi et al., 1993; He and MacLeod, 1996; Smallman et al., 1996), but these studies have not determined whether convergence occurs in the retina or cortex.

In this paper we characterize physiological convergence by measuring the responses of single parvocellular lateral geniculate nucleus (LGN) cells to high spatial frequency interference fringes over a range of orientations. The optics of the eye blur the images of fine patterns before they reach the retina and obliterate nearly all of the contrast at spatial frequencies $>60$ cycles $^{\circ}\left(\mathrm{c} /{ }^{\circ}\right)(\mathrm{Camp}-$ bell and Gubisch, 1966; Williams et al., 1994). Because the neural connection patterns that we would like to examine can be probed only with stimuli of this fine spatial scale, we use interference fringes to bypass the optics and form gratings directly on the retina.

We hypothesize possible underlying circuits by comparing our spatial frequency data with the frequency responses of receptive field models that estimate the number, configuration, and weights of cones that feed the centers and surrounds. Some cells produced responses consistent with a single-cone excitatory center. However, the majority of cells, although responding well to very high spatial frequencies, produced irregular spatial frequency-response curves that indicate input principally from a single cone together with weak input from nearby cones.

Part of this project has been presented previously in abstract form (McMahon et al., 1995). 


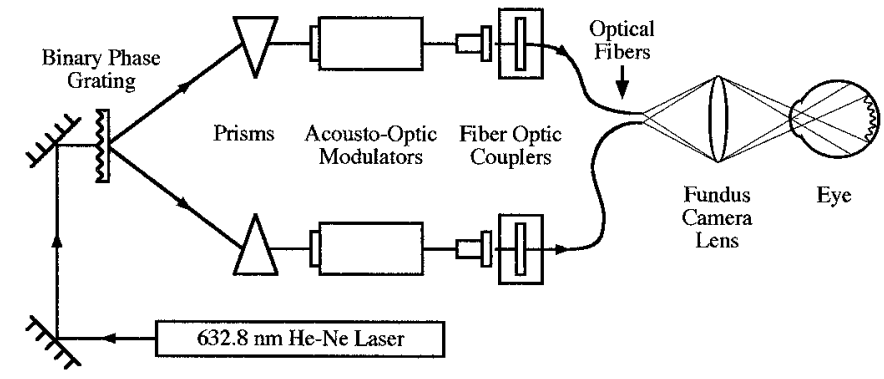

Figure 1. Fiber optic interferometer. The light from a Helium-Neon laser is split into two equal beams by a binary phase grating and directed through acousto-optic modulators, which control the interference fringe contrast and drift rate. The light is then coupled into polarizationpreserving single-mode optical fibers. The fiber tips produce mutually coherent outputs that are used as the interferometric point sources. The separation and orientation of the fiber tips are manipulated by computercontrolled actuators to vary the spatial frequency and orientation of the interference fringe, respectively. The fiber tips are mounted on top of a modified fundus camera and are imaged through the fundus camera lens into the pupil plane of the monkey's eye.

\section{MATERIALS AND METHODS}

Visual stimulation. We constructed a fiber optic interferometer (Fig. 1) that projects laser interference fringes through a modified fundus camera onto the retina of a macaque monkey held in a stereotaxic apparatus. The light from a Helium-Neon laser is split into two equal beams by a binary phase grating and directed through two acousto-optic modulators (AOMs) that control whether the beams are on or off. After emerging from the AOMs, the beams are coupled into polarization-preserving single-mode optical fibers. The fiber tips produce mutually coherent outputs that are used as the interferometric point sources. These point sources are imaged by the fundus camera in the pupil plane of the eye and interfere with each other to produce a sinusoidal intensity pattern on the retina. The interference fringe has a wavelength of $632.8 \mathrm{~nm}$, covers $30^{\circ}$ of visual angle, and has a retinal illuminance of 600 trolands at the center of the field. The use of optical fibers as interferometric point sources dispenses with the need for additional optical components except one mirror and the fundus camera lens. This compact design permits the interferometer to be used in conjunction with a physiological recording setup.

The fundus camera is mounted on an adjustable goniometer and is positioned so that the images of the point sources in the pupil lie at the center of rotation of the device. A mirror within the fundus camera can be positioned either to project the interference fringe onto the retina or to allow the experimenter to view the monkey's retina. During manual receptive field mapping, the fundus camera is positioned to the side, and the interferometer beams are blocked. Reverse-projected images from the fundus camera are used to map the position of the foveas and to center the interference fringe over the receptive field. Fringe spatial frequency, orientation, contrast, and drift rate are controlled by a Macintosh computer.

Grating contrast and drift rate. The AOMs chop the light beams into a train of $1 \mathrm{msec}$ rectangular pulses separated by $1 \mathrm{msec}$. This underlying pulse rate of $500 \mathrm{~Hz}$ is much too fast for the photoreceptors to track. To vary the fringe contrast, the relative temporal phase of the pulses is manipulated by computer control of the AOMs. When the pulses from the two beams are presented simultaneously (with complete temporal overlap) they interfere and a grating of $100 \%$ contrast is produced. When the pulses are alternated, so that one is off whenever the other is on, interference is not possible, and a zero contrast grating is formed. This procedure allows the Michelson contrast to be varied from 0 to $100 \%$ while maintaining a constant space-averaged retinal illuminance. Previous work has shown that this technique allows precise control of interference fringe contrast (Williams, 1985a; MacLeod et al., 1992).

The AOMs are also used to control the spatial phase of the interference fringe. The phase difference between the $40 \mathrm{MHz}$ radio-frequency signals that drive the AOMs produces a delay in one of the wavefronts relative to the other. This delay causes a shift in the spatial phase of the interference fringe. To produce a drifting interference fringe, the phase shift is updated every $2 \mathrm{msec}(500 \mathrm{~Hz})$, which permits smooth and stable drift at any temporal frequency within the visible range.
Spatial frequency and orientation. The fringe spatial frequency, $f$ (cycles per degree), is proportional to the separation, $d$ (in millimeters), between the two point sources in the pupil plane and is represented by $f=$ $\pi d / 180 \lambda$, where $\lambda$ (in millimeters) is the wavelength of the laser source. The fringe orientation is perpendicular to the orientation of the two point sources. The separation and orientation of the fiber tips (which are the point sources) are manipulated by computer-controlled actuators so that they move symmetrically about the center of the pupil. The actuators are equipped with optical encoders to ensure that the requested and actual positions of the motors coincide. The relationship between tip positions and spatial frequency was measured by imaging the interferometric point sources with a high-resolution CCD camera. These measurements were used to produce a look-up table that was used during experiments. The average difference between the requested and measured spatial frequencies in a subsequent calibration was $1.9 \mathrm{c} /{ }^{\circ}$, which is small compared to the very high spatial frequencies of interest here.

Fringe stability. Interferometers in which the interfering beams have separated optical paths can be vulnerable to fringe instability caused by vibration, which produces path length differences between the two beams. To lessen this problem, the interferometer was mounted on a $0.5 \times 0.6 \mathrm{~m}$ vibration-isolating table, and the number of optical components and optical path length before the optical fibers were minimized. However, the use of polarization-preserving single-mode optical fibers is a source of fringe instability because small changes in the position or bending of the fibers will alter the phase of the emerging wavefronts. Although the spatial phase of the fringe is controlled precisely by the AOMs within a trial (see "Grating contrast and drift" rate above), small differences in the absolute position of the fibers (but not their tips) between trials prevent us from knowing the spatial phase relationship between fringes presented on different occasions with certainty. However, even were the spatial phase of the fringe known with certainty, pulse and respiratory artifacts move the eyes (in the paralyzed animal) by amounts that can cause substantial changes in the spatial phases of high spatial frequency gratings. These small movements cannot be obliterated even by anchoring the eye. Our analysis therefore does not make use of information about spatial phase.

Preparation. The experiments were conducted on a $6.1 \mathrm{~kg}$ male Macaca nemestrina and three Macaca fasicularis (two females, 3.3 and $2.8 \mathrm{~kg}$, and a $3.8 \mathrm{~kg}$ male). Each animal was anesthetized initially with ketamine hydrochloride. Indwelling cannulas were inserted in the saphenous veins, and the remaining surgery was completed under sufentanil citrate anesthesia. The animal's head was then mounted in a stereotaxic head holder, and a craniotomy was made above the thalamus. Electrodes were attached to the skull to monitor EEG, and two electrodes were placed in the arms to monitor ECG. No procedure (other than the initial injection) was undertaken without anesthesia. After surgery the animal was given a continuous infusion of sufentanil citrate in lactated Ringer's solution during a $3 \mathrm{hr}$ observation period to assess the adequacy of anesthesia. Initially the dose was $3 \mu \mathrm{g} \cdot \mathrm{kg}^{-1} \cdot \mathrm{hr}^{-1}$, and was increased if there were any signs of arousal. After the adequacy of anesthesia had been established, the animal was given a continuous infusion of sufentanil citrate and vercuronium bromide $\left(100 \mu \mathrm{g} \cdot \mathrm{kg}^{-1} \cdot \mathrm{hr}^{-1}\right)$ to immobilize the eyes. EEG and ECG were monitored continuously, and any signs of arousal were corrected by increasing the rate of anesthetic infusion. The animal was ventilated with a respirator at a rate and tidal volume that kept end-tidal $\mathrm{CO}_{2}$ close to $4 \%$. The body was wrapped loosely in a heating blanket that was controlled by a subscapular thermistor. The pupils were dilated with atropine sulfate, and the corneas were protected with gaspermeable contact lenses chosen to correct the animal's refraction, based on hand-held ophthalmoscopy. Every $24 \mathrm{hr}$ the lenses were removed, and the eyes closed for at least $4 \mathrm{hr}$. The corneas and lenses were periodically examined, and the experiment was aborted if any clouding of the optical elements was observed. We rarely saw signs of physical deterioration during the experiment. At the end of the experiment the animal was given an overdose of sodium pentobarbital and perfused through the heart. All animal procedures used were in accordance with the most recent guidelines published by the National Institutes of Health (1994).

Identification of cells. At the beginning of the experiment, and every few hours thereafter, the positions of the foveas were established with the fundus camera and reverse-projected onto a tangent screen $1.5 \mathrm{~m}$ in front of the animal. Single neurons were targeted in the parvocellular layers of the posterior LGN where receptive fields lay within $5^{\circ}$ of the foveal center. They were identified on the basis of their relatively poor contrast and flicker response (in comparison to magnocellular LGN cells) and by their laminar location. Their receptive fields were manually mapped onto 


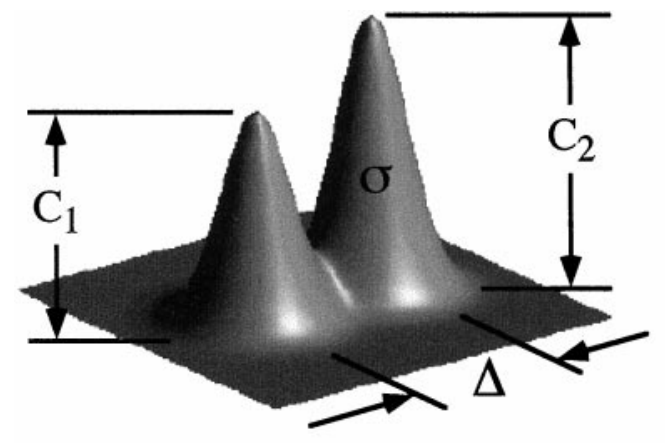

Model Parameters

$\mathrm{C}_{1}$ cone weight one (arbitrary units)

$\mathrm{C}_{2}$ cone weight two (arbitrary units)

$\Delta$ cone spacing (degrees of visual angle)

$\sigma$ cone aperture (degrees of visual angle)

( Gaussian $\sigma=$ Gaussian radius $/ \sqrt{ } 2$ )

$\theta$ orientation of cones (degrees)

\section{Fourier Transform $\downarrow$ \\ Frequency Response, $|F(u, v)|$}

Figure 2. Model receptive field with one- or two-cone center. The spatial form of the model is illustrated pictorially in the top of the figure. The Fourier transform of the analytical expression of the model can be expressed in terms of the spatial model parameters. The amplitude of this complex function represents the frequency response of the model. This function, $|F(u, v)|$, was fit to the full 2-D spatial frequency data set for a given cell to obtain the best estimates of the spatial model parameters. the tangent screen before the interferometer was positioned in front of the animal. The large field filled by the interference fringe was centered on each receptive field and always covered, and extended well beyond, the receptive field under study.

Physiological recording. Action potentials were recorded with glassinsulated tungsten microelectrodes from as many parvocellular LGN neurons as possible for up to $72 \mathrm{hr}$ (less if the cells were unresponsive or the recordings were unstable). Spike times were recorded to the nearest $1 \mathrm{msec}$ and saved by the computer that controlled the interferometer. This also provided real-time analysis of responses.

Once a cell was identified as a parvocellular neuron and its receptive field was mapped, we obtained finely sampled spatial frequency-response curves. The number of orientations sampled depended on the length of time we were able to hold a cell and the quality of its spikes. When cells could be held long enough, we made further coarsely sampled measurements at other spatial frequencies and orientations in an attempt to tile the two-dimensional (2-D) spatial frequency domain. Measurements of spatial frequency response for a single grating orientation provide a radial slice in the 2-D spatial frequency plane. All spatial frequencytuning curves were measured with $100 \%$ contrast interference fringes.

For each measurement, action potentials were recorded while an interference fringe drifted steadily across the receptive field at $10 \mathrm{~Hz}$ for 2 sec. Gratings of different spatial frequencies and orientations were presented in random order.

Analysis of responses. The first Fourier component was taken as the response amplitude and was calculated as the peak of the function resulting from the convolution of the response spike train from a given presentation and a $10 \mathrm{~Hz}$ sinusoid. This was done for each response sample and then the amplitudes were averaged, as opposed to the traditional method of averaging the response samples before computing the amplitude. This was necessary because small movements of the eye and trial to trial differences in fringe phase prevented us from knowing the absolute phase of the response (see "Fringe stability" above). The variations across trials in absolute phase of each response can result in the obliteration of the responses if they are averaged before computing the amplitude. Final data points are the average of at least 20 presentations (sometimes less if the recording was lost during a block). Error bars in all graphs represent \pm 1 SEM.

We neglected the higher order harmonics in the responses to our sinusoidal stimulation. These higher harmonics have been shown to be small and to mainly influence the overall level (and not the shape) of the spatial frequency-response profiles (Thibos and Levick, 1983).

Computer modeling of cellular response. The goal was to assess the compatibility of the measured spatial frequency responses with a one- or two-cone center receptive field model. This model has a small number of parameters that represent the cone weights, cone spacing, cone aperture, and the spatial orientation of the two cones (Fig. 2). The cone light gathering aperture is represented by a Gaussian function with $\sigma$ equal to
0.204 times the cone inner segment diameter (Chen et al., 1993). The spatial profile of the model can be expressed analytically. The frequency response of any spatial detector array is given by the 2-D Fourier transform of the array (Bracewell, 1986). By taking the Fourier transform of the spatial receptive field, we can express the spatial frequency response of the model as a function of the spatial model parameters. The model assumes that a cell behaves as a linear spatial filter. This appears to be true of parvocellular cells in the LGN (Kaplan and Shapley, 1982; Derrington and Lennie, 1984). Retinal ganglion and LGN cell receptive fields have traditionally been modeled by a difference of Gaussians function with a sensitive, compact Gaussian center and an insensitive, spatially extended Gaussian surround (for review, see Walraven et al., 1990). Because our objective was to understand the fine structure of the receptive field center, our model does not include a spatially extensive inhibitory surround and therefore produces no decrease in response at low spatial frequencies (Enroth-Cugell and Robson, 1966). For this reason, we excluded data collected at low frequencies $\left(<10 \mathrm{c} /{ }^{\circ}\right)$ when fitting the model. Further consequences of a spatially extended inhibitory surround are examined in the Results section. We subtracted the nonzero response amplitude generated by spontaneous activity during zero contrast conditions from all measurements before fitting. The model is static and ignores the temporal response properties.

To obtain the best estimate of the model parameters, a weighted least squares procedure was used to fit the model to the full 2-D spatial frequency data set of each cell. We used both quasi-Newton and simplex search algorithms and chose a wide range of initial parameter values to ensure that the global minimum of the error function was found. The resulting parameter estimates could then be compared to anatomical and psychophysical measurements of cone aperture and spacing. This general technique of reconstructing spatial receptive fields from 2-D spatial frequency responses has been used previously to infer the spatial structure of cat retinal ganglion cell and LGN receptive fields (Thibos and Levick, 1983; Soodak, 1986; Soodak et al., 1987, 1991).

If the one- or two-cone center model provided a poor fit to the data gathered or produced wildly incorrect estimates of the physiological parameters, we used more elaborate models that included complex center and/or surround arrangements. The calculations were performed as follows. The cone center positions for a 2-D photoreceptor mosaic image were located with an image-processing algorithm (NIH Image, http:// rsb.info.nih.gov/nih-image) and stored in an array. Synthetic receptive fields were created by assigning various weights to the cone positions in the array. We assumed that all cones were equally sensitive and that all cone signals were summed linearly. This array was convolved with the Gaussian that represented the cone aperture function for that eccentricity. An image-processing program (IPLab, Signal Analytics) was then used to calculate the 2-D frequency response of the constructed receptive field. Qualitative features of the resulting spatial frequency-response profiles were then compared with the experimentally obtained responses. 
Figure 3. Simulated receptive fields and their frequency responses for interference fringes and conventionally imaged gratings. The left column shows three simple receptive field profiles comprised of either one or two foveal cones. The cone lightgathering aperture is best approximated by a Gaussian with $\sigma$ equal to 0.204 times the cone inner segment diameter (Chen et al., 1993). A cone inner segment diameter of $2.3 \mu \mathrm{m}$ (or $0.01^{\circ}$ ) is used. The right two columns show the frequency-response characteristics of the receptive fields for interferometric and conventionally imaged stimuli plotted to $s f_{\mathrm{x}}$ and $s f_{\mathrm{y}}=120 \mathrm{c} /{ }^{\circ}$. This is calculated by taking the Fourier transforms of the spatial receptive field profiles. However, to obtain the frequency response for conventional stimuli, we must also multiply the frequency response by the modulation transfer function of the eye (Williams et al., 1994). In the interferometric case, the three different receptive fields could easily be distinguished by measuring spatial frequency-tuning curves at a number of orientations. These curves would correspond to single slices in the 2 -D spatial frequency plane (two are shown in the center column). The receptive field configurations could not be differentiated by conventionally measured spatial frequency responses because optical blurring obliterates the contrast of the requisite high spatial frequencies.

\section{Preliminary modeling}

We used the simple one- or two-cone center model with recent measurements of the optical quality of the eye (Williams et al., 1994) and the size and shape of the cone photoreceptor lightcollecting aperture (Chen et al., 1993) to produce plots of the 2-D frequency response of hypothetical LGN cells to conventionally viewed gratings and to gratings produced by laser interference. The results are graphically presented in Figure 3 for the three retinal receptive field profiles shown in the left column.

The frequency responses of the receptive fields for interference fringe stimuli are shown in the center column. The radial symmetry of the top plot illustrates that the response of a single cone does not depend on orientation and will decline smoothly across spatial frequency. In the two-cone cases, gratings oriented along the axis connecting the cones will be detected as if seen by a single cone and will produce the characteristic smooth decline in frequency response. Gratings perpendicular to the axis connecting the cones will show a series of response zeros. These occur at spatial frequencies that stimulate one cone with a dark bar and the other cone with a light bar.

The right column of plots in Figure 3 shows the frequency response after taking into account the blurring of the stimulus by the optics of the eye (Williams et al., 1994). It is clear from a comparison of this with the response profiles in the center column that the irregularities in spatial frequency response that are necessary to distinguish these different receptive field profiles are obliterated when gratings are imaged by the optics of the eye. When interference fringes are used, there is no degradation of stimulus contrast at high spatial frequencies, and the different receptive field organizations are readily distinguished by measuring spatial frequency responses at different orientations.

\section{RESULTS}

\section{Calculated Frequency Responses}
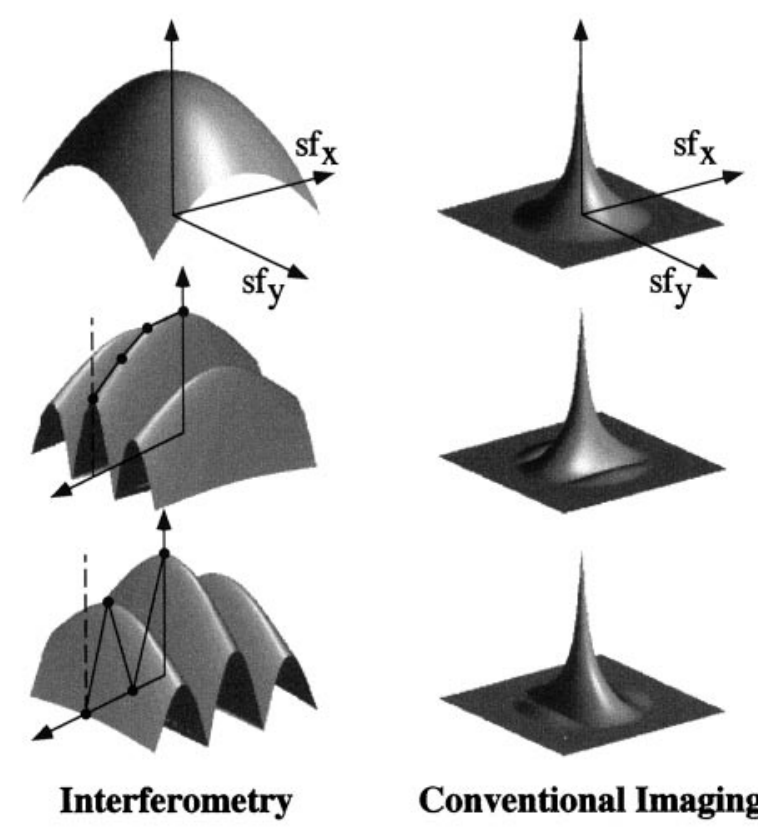

Conventional Imaging

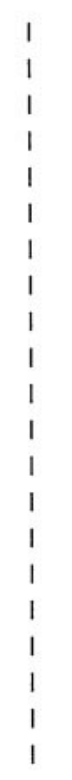

\section{Cellular responses and modeling}

We recorded from 71 parvocellular LGN cells with receptive fields within $5^{\circ}$ of the foveal center. Sixty of 71 cells produced reliable spatial frequency responses at or beyond $100 \mathrm{c} /{ }^{\circ}$. Figure $4 a$ shows an example. Figure $4 b$ shows a contrast response curve measured at $100 \mathrm{c} /{ }^{\circ}$ for the same cell. Figure 4, $c$ and $d$, show counterpart curves for another cell; in this case the contrast response function was measured at $120 \mathrm{c} /{ }^{\circ}$. The highest spatial frequency responses reported using conventional physiological techniques have been in the range of $40 \mathrm{c} /{ }^{\circ}$ in parvocellular LGN cells (Derrington and Lennie, 1984).

We obtained measurements at two or more orientations from 47 cells; for a further 24 we were only able to obtain measurements at a single orientation. For those sets of spatial frequency responses to which we could apply the one- or two- cone model, only one was adequately fit ( $>90 \%$ of the variance accounted for). This cell, whose response profiles are shown in Figure 5, was well fit by a two-cone center. The other 46 cells had flat or bumpy spatial frequency-response functions that could not be adequately fit with our model. Four examples are shown in Figure 6. The model accounted for an average of $65 \%$ of the variance in the responses of the 47 cells.

The perturbations in the spatial frequency-response curves are consistent attributes of cells, as one can see by comparing repeated measurements on the same cell. Figure $7 a$ shows two spatial frequency-tuning curves measured for a cell $1 \mathrm{hr}$ and 20 min apart. Figure $7 b$ shows corresponding curves for another cell,measured $15 \mathrm{~min}$ apart. The measurements for this cell were also made at slightly different spatial frequencies. If optical irregularities in the cornea or lens were causing significant alterations in the response of the cell, then slightly varying the positions of the point sources in the pupil plane (by making measurements at 

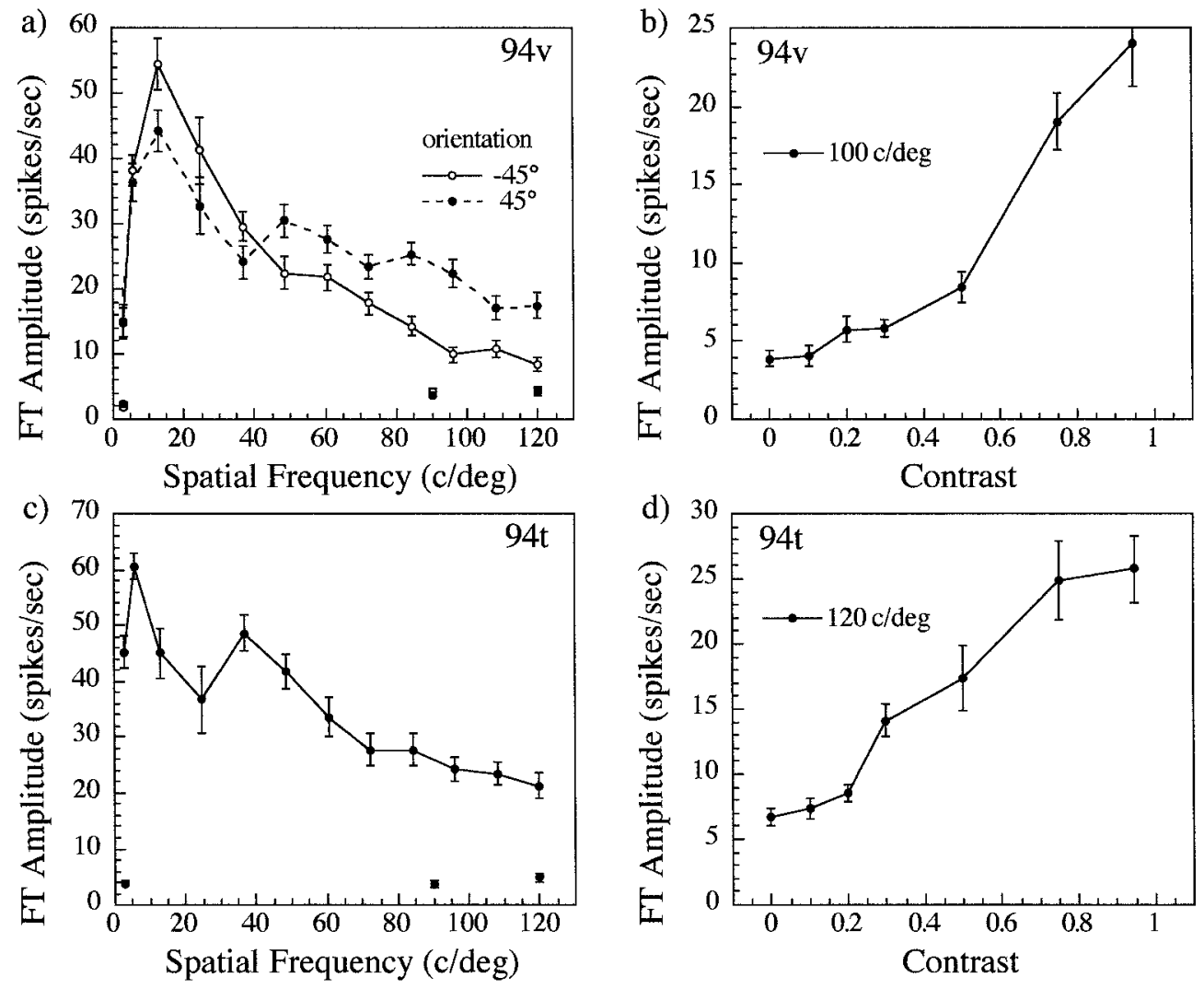

Figure 4. a, Spatial frequency-tuning curves for a single neuron $(94 \mathrm{v})$ measured at $-45^{\circ}$ and $+45^{\circ}$ orientation. $b$, A contrast-response curve for the same neuron, measured at $100 \mathrm{c} /{ }^{\circ}$. $c$, Spatial frequency-tuning curve and contrastresponse curve $(d)$ measured at $120 \mathrm{c} /{ }^{\circ}$ for another cell (94t). Both receptive fields were $0.9^{\circ}$ from the fovea. The unconnected data points were measured at zero contrast to assess baseline response amplitude. Error bars in these and in subsequent plots represent \pm 1 SEM.

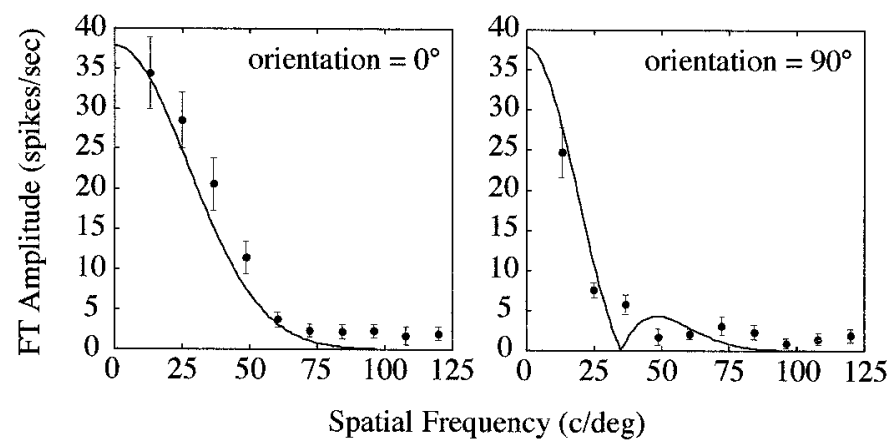

Figure 5. Fit of the one- or two-cone center model to the measured spatial frequency-response curve. The receptive field of the cell was $1.0^{\circ}$ from the fovea. The baseline response amplitude has been subtracted, and data collected at $<10 \mathrm{c} /{ }^{\circ}$ are excluded. The cell is best fit by a two-cone center with model parameters $c_{1}=37.8, c_{2}=37.8, \Delta=0.015^{\circ}, \sigma=0.010^{\circ}$, $\theta=270^{\circ}$. The model fit accounts for $96 \%$ of the variance; cell $97 \mathrm{k}$.

slightly different spatial frequencies) should cause significant changes in the shape of the spatial frequency-tuning curve. This is not the case, suggesting that local optical irregularities are not the cause of the irregular responses. Repeated measurements were conducted on nine other cells. For all of these cells, the shapes of the spatial frequency-tuning curves were very similar, even if the overall response magnitude varied slowly over time. It is clear from the poor fits of the one- or two-cone center model that it does not adequately describe the underlying spatial profiles of the receptive fields. In the following paragraphs we explore possible reasons.

The irregular shape of the spatial frequency-response curves might be caused by the cells being overdriven by a high contrasthigh spatial frequency stimulus that they have never before been exposed to. Previous workers have shown that primate parvocellular LGN neurons show a much more gradual increase in response with increasing contrast than magnocellular neurons (Shapley et al., 1981; Kaplan and Shapley, 1982). This response is roughly linear at low contrasts, with a mild saturation at high contrasts. Contrast response curves measured for cells at spatial frequencies $>100 \mathrm{c} /{ }^{\circ}$ (Fig. $4 b, d$ ) showed a similar relationship between contrast and response to that seen in parvocellular neurons at low spatial frequencies, ruling out response saturation as the cause of the irregular shapes of the spatial frequencytuning curves.

Retinal photoreceptors act as waveguides (Enoch and Tobey, 1981). While current waveguide theories predict smooth and symmetric spatial frequency-tuning curves (Pask and Stacey, 1998), it is conceivable that individual cones have idiosyncratic tuning curves for coherent interference fringes. To examine the effect of waveguide properties on cellular responses, we performed a control experiment that varied the positions of the point sources in the pupil plane. Measurements were made for centered point sources and then re-measured with the point sources displaced by $1 \mathrm{~mm}$ in various directions in the pupil plane. This displacement would produce an $\sim 5^{\circ}$ change in the angle of the incident wavefronts that form the interference fringe on the retina. Two cells tested in this way showed little deviation between the centered and decentered point source conditions, suggesting that receptor waveguide properties do not cause the irregular shape of the response (Fig. 8). It is, however, difficult to completely rule out this possibility.

Our model takes no account of any spatially extensive Gaussian surround. The main effect of a large Gaussian surround is to produce a decrease in response to low spatial frequencies (Enroth-Cugell and Robson, 1966). In the following paragraphs 
Figure 6. Examples of cells whose receptive fields are poorly characterized by the one- or two-cone center model. Each panel shows, for a different cell, spatial frequency-response curves measured at two orientations. The model fit accounted for $57,52,46$, and $76 \%$ of the variance of the responses of $93 \mathrm{v}$, 93aa, $94 \mathrm{~b}$, and $104 \mathrm{r}$, respectively. All but one cell (that of Fig. 5) produced spatial frequency-response profiles that were poorly fit by the one- or two-cone center model (46 of 47). The average variance accounted for was $65 \%$ for the 47 cells fit by the model.

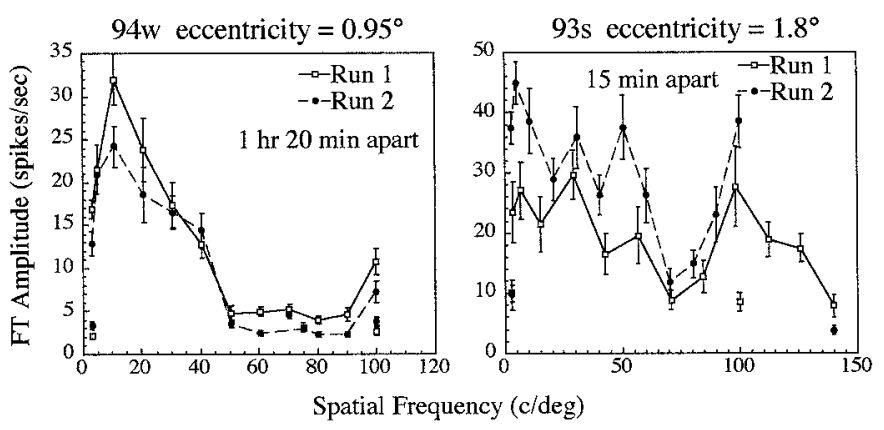

Figure 7. Reproducibility of the measured spatial frequency-response curves. Repeated sets of measurements for cell $94 \mathrm{w}$, collected $1 \mathrm{hr}$ and 20 min apart, and for cell 93s, collected 15 min apart. The spatial frequency sampling of the two sets is slightly different for $93 \mathrm{sec}$, which results in different pupil entry points of the point sources. The repeated curves are in good agreement despite the time and spatial frequency sampling differences.

we rule out three potential sources of the observed irregular high spatial frequency responses: nonuniform synaptic weights in the surround, aliasing within the surround, and small surrounds.

To examine the effects of nonuniform cone weights in the surround, a synthetic foveal LGN cell was constructed with a single-cone excitatory center and a spatially extensive inhibitory surround. The initial weights of cones in the surround were calculated using the best fitting Gaussian parameters for Croner and Kaplan's (1995) 0-5 $5^{\circ}$-cell population after correction for the monkey's optical contrast attenuation (peak sensitivity, $k_{\mathrm{S}}=$ 8.6; Gaussian radius, $r_{\mathrm{S}}=0.16^{\circ}$ ). Each surround cone weight was then multiplied by a number drawn randomly from a uniform distribution between 0.5 and 1.5. The 2-D spatial frequency
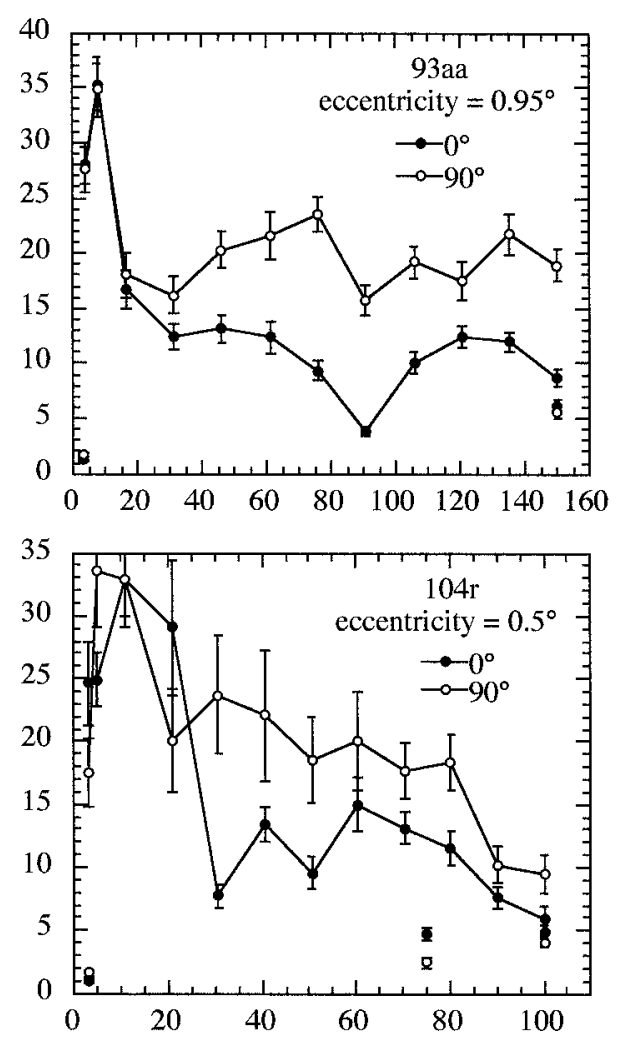

\section{Spatial Frequency (c/deg)}

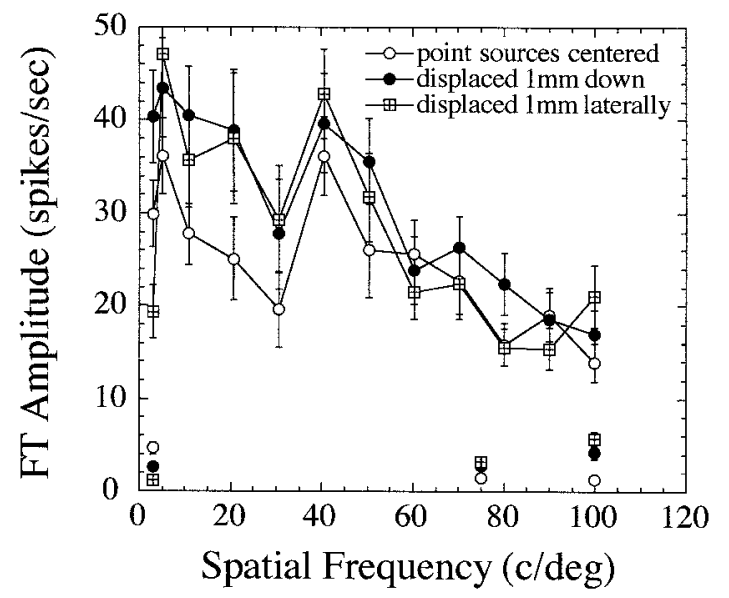

Figure 8. Displacement of the point sources does not alter the response. Spatial frequency-tuning curves for cell $104 \mathrm{q}\left(\right.$ eccentricity $\left.=0.2^{\circ}\right)$ measured with the interferometric point sources centered and then remeasured with the point sources displaced by $1 \mathrm{~mm}$ in various directions in the pupil plane. This change in the angle of the incident wavefronts does not change the irregular shape of the spatial frequency-response, suggesting that receptor waveguide properties do not underlie the irregular response.

response for the synthetic cell was then calculated by taking the Fourier transform of the receptive field function. Four radial slices through the spatial frequency plane were then derived (corresponding to spatial frequency-response curves for four different grating orientations). The results are shown in Figure 9. Varying the individual cone weights in the surround by $\pm 50 \%$ has almost no effect on the response of the cell. 


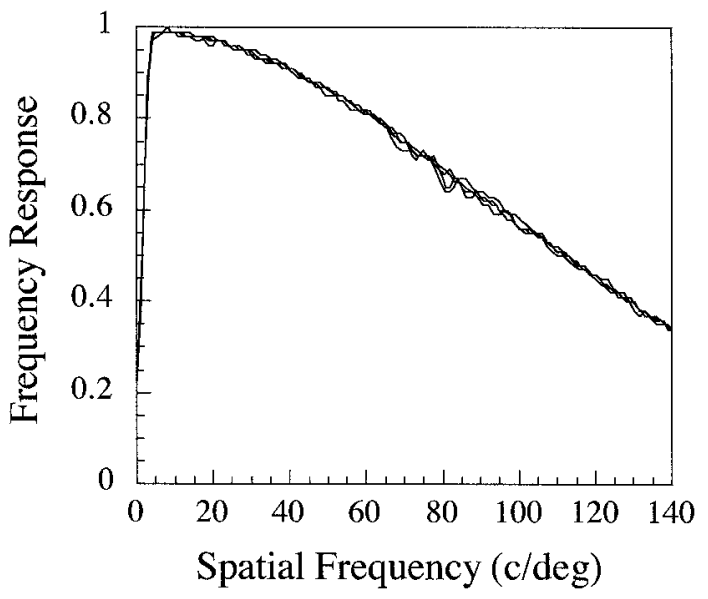

Figure 9. Frequency response of a synthetic cell with nonuniform cone weights. The cell is constructed from a single-cone center and a spatially extensive Gaussian inhibitory surround. The cone weights in the surround are randomly varied between plus and minus $50 \%$ of their original value. The plot shows four slices through the 2-D frequency response of the cell. Severe perturbation of the cone weights in the surround has little effect on the response.

Because our interferometric technique allows us to produce stimuli finer than the photoreceptor mosaic, it is necessary to examine the consequences of undersampling by the array of cones in the surround. Undersampling by an array can produce spurious low-frequency components in the sampled signal of fine patterns (aliasing). Aliasing occurs when the spatial frequency of the stimulus exceeds one-half the sampling frequency of the array (the Nyquist frequency). It has previously been shown that receptor disarray causes the high spatial frequency aliasing responses to be of lower magnitude and spread over a wider frequency range (Yellott, 1983; Williams, 1985a; He and MacLeod, 1996). To evaluate the possibility that the high spatial frequency responses of the cells are caused by aliasing in the surround, we examined the effects of surround size and receptor disarray on simulated surround responses.

We used surround size estimates from Croner and Kaplan's (1995) sample of retinal P-cells between 0 and $5^{\circ}$ eccentricity after correction for the monkey's optical contrast attenuation (Gaussian surround, median $r_{\mathrm{S}}=0.16^{\circ}$, interquartile range $=$ $0.07^{\circ}$ ). We created surrounds spanning this size range from a photoreceptor array image centered at $4^{\circ}$ eccentricity (Liang et al., 1997; see Fig. 11b) and from a foveal image of a macaque retinal whole mount (Williams et al., 1991). We used real photoreceptor mosaic images to ensure realistic amounts of receptor disarray, which generally increases with retinal eccentricity (Hirsch and Miller, 1987). Gaussian surrounds were constructed from these receptor arrays with radius equal to median plus interquartile range and median minus interquartile range (Croner and Kaplan, 1995), and their frequency responses were calculated. Four slices were taken in the 2-D spatial frequency plane $\left(45^{\circ}\right.$ apart) for each of the surround frequency responses. These slices correspond to spatial frequency-tuning curves measured for four different grating orientations in the spatial domain. Figure 10 shows frequency-response curves for the large and small foveal and $4^{\circ}$ surrounds (curves labeled "s"). The surrounds respond mainly at very low spatial frequencies $\left(<10 \mathrm{c} /{ }^{\circ}\right)$, with a slight bump around the sampling frequency of the mosaics $\left(80 \mathrm{c} /{ }^{\circ}\right.$ for the foveal mosaic and $46 \mathrm{c} /{ }^{\circ}$ for the $4^{\circ}$ mosaic). This modeling

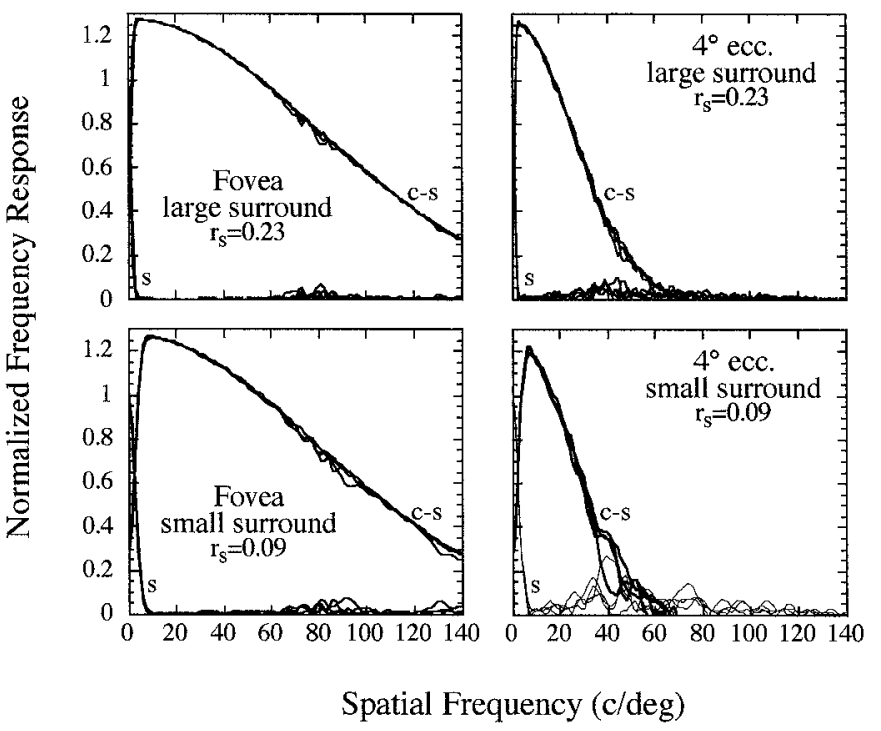

Figure 10. Frequency responses of synthetic receptive fields with Gaussian surrounds. Frequency responses are calculated for large and small Gaussian surrounds constructed from a foveal cone mosaic and one at $4^{\circ}$ eccentricity. The frequency response of each surround is a 2-D function. The four curves labeled $s$ in each plot represent spatial frequency-tuning curves (or slices through 2-D spatial frequency space) for orientations separated by $45^{\circ}$. The surrounds respond mainly at low spatial frequencies $\left(<10 \mathrm{c} /{ }^{\circ}\right)$, but a small response is generated around the sampling frequency of each mosaic ( $46 \mathrm{c} /{ }^{\circ}$ for the $4^{\circ}$ and $80 \mathrm{c} /{ }^{\circ}$ for the foveal mosaic). Curves labeled $c-s$ show frequency responses of receptive fields with a single-cone center and the constructed inhibitory surrounds. The surrounds produce mainly a low-frequency cutoff.

shows that the inhibitory contribution of the surround to the response of a cell would cause mainly a low spatial frequency cutoff. The high-frequency components increase in size as the surround becomes smaller and collects from fewer cones and are most prominent for the small surround at $4^{\circ}$ eccentricity, where the maximal high spatial frequency response is $33 \%$ of the peak response of the surround. Each panel of Figure 10 also shows frequency-response curves of a receptive field constructed from a single-cone center and the previously discussed surround (labeled "c-s"). The integrated sensitivity of the center was set to be $28 \%$ greater than the surround-the average amount found by Croner and Kaplan (1995). The surround contributes only very slight irregularities to the overall curve, and we conclude that aliasing within the surround cannot be the source of the irregular response profiles observed at high spatial frequencies.

It has previously been noted that parvocellular LGN cells show considerable variation in the form of the contrast sensitivity function at low spatial frequencies (Derrington and Lennie, 1984). This variability results in a wide range of estimates for the size and strength of the inhibitory surround. To assess the relationship between surround size and high spatial frequency response, synthetic cells were constructed with a single-cone center and Gaussian inhibitory surrounds of several small sizes. Spatial frequency-tuning curves were generated in the same manner as described above. The results are shown in Figure 11. The traditional LGN cell inhibitory surround would have to be much smaller than surrounds that have been previously measured before it would exert any effect other than a low-frequency cutoff. Because the foveal cone spacing is $\sim 0.01^{\circ}$ (Packer et al., 1989), surrounds with sizes of this order would collect signals from a small number of cones. 

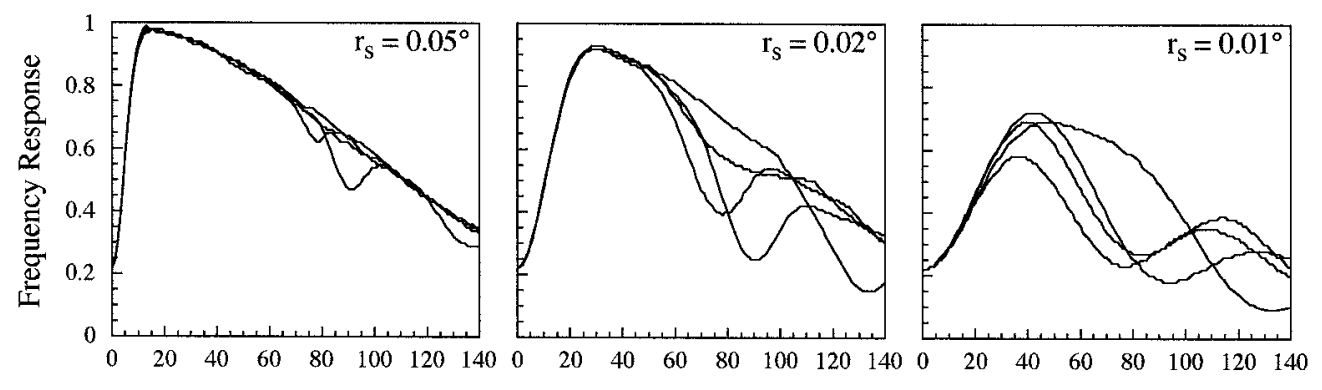

Spatial Frequency (c/deg)

Figure 11. The effect of small inhibitory surrounds on single-cone frequency response. Each plot shows four slices of the 2-D frequency response of a synthetic receptive field with a single-cone center and a Gaussian inhibitory surround of the specified size. The surrounds were assigned a relative sensitivity $\left(k_{\mathrm{S}} r_{\mathrm{S}}{ }^{2} / k_{\mathrm{C}} r_{\mathrm{C}}{ }^{2}\right)$ of 0.78 . To produce significant effects other than a low-frequency cutoff, the surround must be very small. Surrounds with $r_{\mathrm{S}}=$ $0.01^{\circ}$ would collect signals from only a small number of cones.

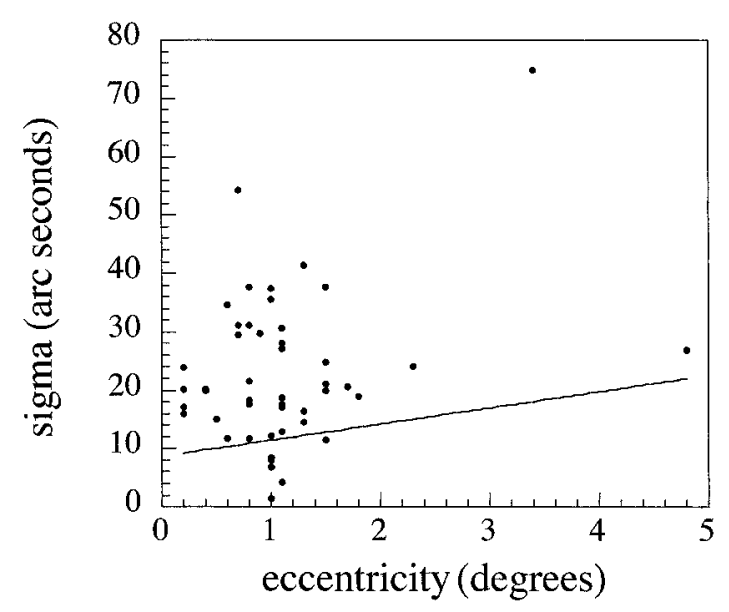

Figure 12. Estimates of Gaussian $\sigma$ for our population. The best-fitting $\sigma$ values for the 47 cells fit with the one- or two-cone center model are plotted together with our best estimate of the physiological cone aperture (see Results). Because $\sigma$ describes the overall falloff of response with increasing spatial frequency, it provides a rough estimate of the underlying spatial extent of the receptive field center. This comparison shows that the receptive field centers of the cells in our population have a $\sigma$ that is larger than that of a single cone.

The most probable account of the perturbations in the measured frequency-response curves is that the center of the receptive field receives its principal input from a single cone-one that defines the overall shape of the curve-with additional weaker inputs from nearby cones. The model parameter $\sigma$ (Fig. 2) provides an estimate of the spatial extent of the receptive field center. This parameter controls the falloff of response with increasing spatial frequency; modulations in the response caused by contributions from other cones take place underneath the response envelope defined by the frequency-response of a single cone. The best fitting $\sigma$ values are plotted in Figure 12 as a function of retinal eccentricity together with the line representing our best estimate of the physiological cone aperture. This estimate is derived from anatomical measurements of cone inner segment diameter in macaque monkeys (Packer et al., 1989) and psychophysical experiments that estimate the effective light collecting aperture of cone photoreceptors as a Gaussian function with $\sigma$ equal to 0.204 times the cone inner segment diameter (Chen et al., 1993). The fact that the fitted $\sigma$ values tend to be larger than the estimated $\sigma$ values for individual cones suggests that the receptive field centers are slightly larger than that of a single cone photoreceptor.

To examine the effects of a center comprised of a principal cone augmented by weak inputs from neighboring cones, receptive fields were constructed from a foveal image of a macaque retinal whole mount (Williams et al., 1991). The receptive fields contain a single cone near the center of the fovea together with varying contributions from the first and second ring of cones that surrounded it. The $0.1 \times 0.1^{\circ}$ mosaic of cones used in these simulations is shown within the first panel of Figure 13. Six receptive fields were constructed; three single-cone receptive fields contain the same sign contribution from their neighbors, and the other three receive an inverted contribution. We varied the contribution of the neighbors from 1 to $20 \%$. For each simulation, every cone in the first ring is given a weight that is a fixed percentage of the center cone. Each cone in the second ring is assigned that same percentage of the value assigned to every cone in the first ring. The frequency responses associated with the receptive fields are shown in Figure 13. This simulation demonstrates that weak input from bordering cones, whether same sign or inverted, can have a profound effect on the high-frequency behavior of a single cone. All cones in this simulation are equally sensitive. (Our exclusion of the $\sim 2.5$ times difference in sensitivity between $\mathrm{L}$ - and $\mathrm{M}$-cones is unlikely to change our conclusion. This is because a very small nearest neighbor contribution is required to cause the observed irregular tuning curves. Therefore, the difference in contribution between the surrounding cones and the center cone is very large compared to the difference between $\mathrm{M}$ - and L-cone sensitivity at $633 \mathrm{~nm}$.)

\section{DISCUSSION}

We have shown that parvocellular LGN cells with receptive fields near the fovea respond reliably to very high spatial frequencies. The fact that most cells respond to frequencies $>100 \mathrm{c} /{ }^{\circ}$ implies that they draw their center input from a very limited area on the retina. Responses to such high spatial frequencies have not previously been seen in vivo because the optics of the eye obliterate the contrast in very fine patterns [in humans, the optics attenuate the contrast in high spatial frequency patterns $\left(>50 \mathrm{c} /{ }^{\circ}\right)$ by $90 \%$ (Williams et al., 1994)]. Derrington and Lennie (1984) reported that macaque parvocellular LGN cells with foveal receptive fields could resolve spatial frequencies up to $40 \mathrm{c} /{ }^{\circ}$. Although Croner and Kaplan (1995) did not display spatial frequency-tuning curves, their macaque parvocellular LGN neurons between 0 and $5^{\circ}$ eccentricity had estimated center sizes similar to those mea- 

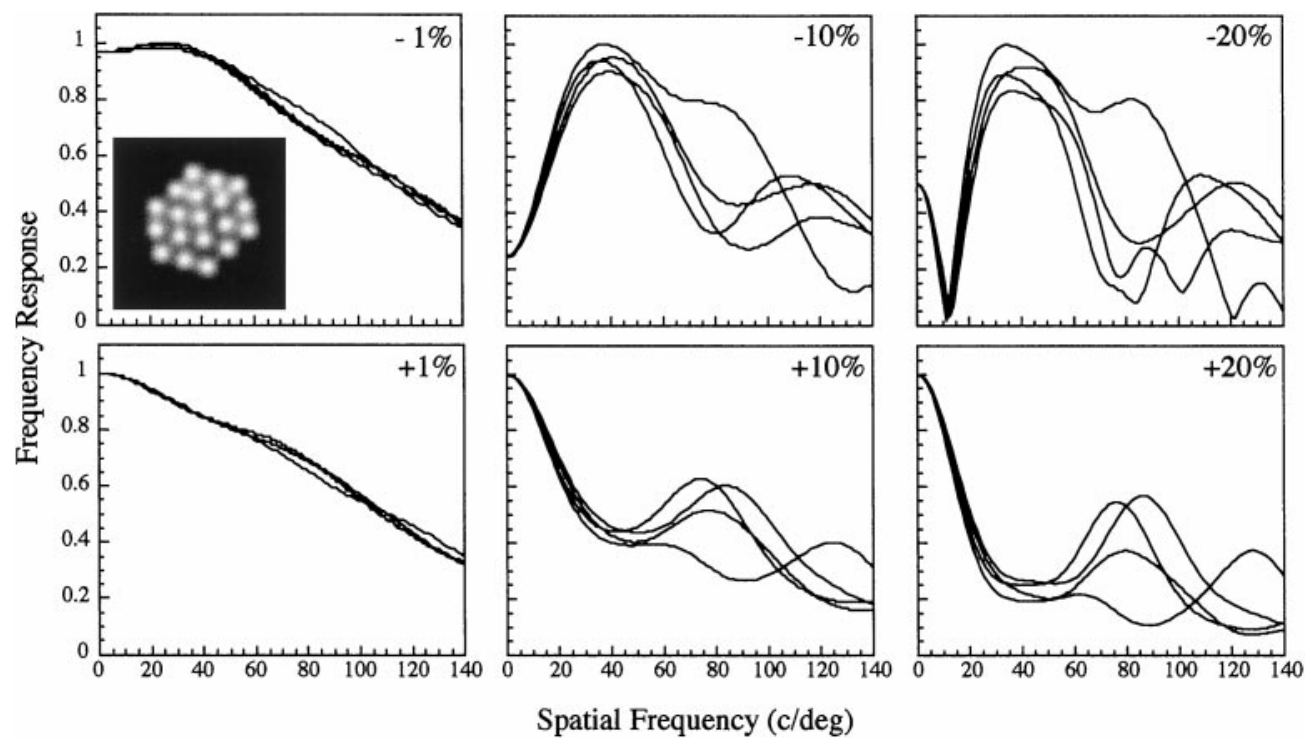

Figure 13. The effect of neighboring cones on the frequency response of a single cone. Each plot shows four slices of the 2-D frequency-response plane of a single cone augmented by various contributions from its nearest neighbor cones. The cone mosaic used is from a macaque retina whole mount and is shown as an inset in the first panel. For each simulation, every cone in the first ring is given a weight that is a fixed percentage of the center cone value. Each cone in the second ring is assigned that same percentage of the value assigned to every cone in the first ring. Simulations are made for single-cone centers with $\pm 1,10$, and $20 \%$ contributions from the nearest neighbors. These simulations demonstrate that a modest contribution from nearby cones, whether same sign or inhibitory, produces large deviations from the smoothly declining single-cone response. sured by Derrington and Lennie (1984). It is difficult to estimate the center size of these small receptive fields from conventional measurements because any small eye movements, errors in the monkey's refraction, or misalignment of the artificial pupils will degrade responses to the already diminished retinal contrast of high spatial frequency stimuli. Deconvolution of these responses with the optical point spread function would then give an artificially inflated estimate of the center size.

Our failure to adequately fit the majority of LGN responses with the single-cone model reveals previously unknown complexities in retinal organization. The irregular spatial frequency responses we measured are consistent with a single-cone center together with weak input from nearby cones. Our modeling shows that qualitatively similar perturbations at high frequencies can be caused by same sign or inhibitory input. Possible sources of weak input from nearby cones are discussed below.

\section{Possible sources of nearest neighbor contribution to the receptive field center}

Gap junction coupling

Primate cone photoreceptor terminals are coupled to each other by gap junctions (Cohen, 1965; Raviola and Gilula, 1973; Tsukamoto et al., 1992). Although the structure of these gap junctions has been studied in detail using electron microscopy, the functional significance is unknown. Functional aspects of receptor coupling have been characterized in lower vertebrates using current injection (for review, see Attwell, 1986), but technical difficulties have prevented similar experiments in primates.

Modeling studies have demonstrated that gap junction coupling can improve contrast sensitivity at low spatial frequencies by averaging the uncorrelated noise between photoreceptors (Tessier-Lavigne and Attwell, 1988). However, cone coupling would decrease contrast sensitivity at high spatial frequencies. This impairment would not be detrimental under normal visual conditions because the optics of the eye already blur the detail in high spatial frequency patterns (Hsu, 1998). Although cone coupling may provide beneficial properties, a number of findings provide evidence against it.

The first line of evidence against functional cone coupling comes from measurements of cone spectral sensitivities. It has been observed that primate cone pedicles contact all of their neighboring pedicles indiscriminately (Raviola and Gilula, 1973; Tsukamoto et al., 1992). The functional spread of electrical activity between cones through gap junctions seems very unlikely because any current spread between cones of different spectral types would significantly shift the $\lambda_{\max }$ values of L- and M-cones toward their average value (Hsu, 1998). However, psychophysically measured cone fundamentals (Stockman et al., 1993) are very similar to suction electrode recordings of isolated cone photoreceptor spectral sensitivities (Baylor et al., 1987), and there is no significant difference between the cone fundamentals measured in dichromatic and trichromatic observers. Measurements of spectral sensitivities of intact cone inner segments also suggest that there is no mixing of cone signals (Schneeweis and Schnapf, 1999).

Psychophysical observations also allow us to push the site of nearest neighbor contribution beyond the level of the cones. When two high-contrast interference fringes of slightly different orientation or spatial frequency are projected onto the retina, observers report the appearance of a distortion product (or difference frequency grating). These difference frequency gratings remain visible when the component gratings that produce them are extremely fine, implying that there is no neural spatial summation between cones prior to the site of the nonlinearity that produces the difference frequency grating (MacLeod et al., 1992). This nonlinearity is most certainly postreceptoral, because voltage recordings from macaque cone outer segments (Schnapf et al., 1990) and inner segments (Schneeweis and Schnapf, 1999) show no significant adaptation at these moderate light levels. Because the difference frequency grating results require no spatial summation prior to the nonlinearity, and the nonlinearity is postreceptoral, these experiments provide further evidence that cone photoreceptors are not functionally coupled to each other. These data also suggest that the contribution from neighboring cones inferred from our measurements originates at a site beyond that of the early sensitivity regulating nonlinearity.

\section{Cellular coupling in the inner retina}

Intracellular Neurobiotin injections have shown that primate parasol ganglion cells are coupled to their nearest neighbors and to amacrine cells (Dacey and Brace, 1992). Although the same study showed no tracer coupling between midget (parvocellular) 
ganglion cells or between midget and amacrine cells, it is possible that coupling via gap junctions is unidirectional (Vaney, 1996) or is modulated by the neurotransmitter and/or light level conditions of the experiment (Baldridge et al., 1998).

\section{Possible sources of localized inhibitory contribution to the receptive field}

In addition to the possible excitatory influence of nearby cones on the receptive field center, our receptive field modeling (Figs. 11, 13) demonstrates that inhibitory contributions substantially smaller in spatial extent than the classical diffuse inhibitory Gaussian surround are a possible source of the irregular responses at high spatial frequencies.

\section{Cone or bipolar cell surrounds}

Cone photoreceptors in nonmammalian retina have antagonistic receptive field surrounds that are generated by synaptic feedback from horizontal cells. This antagonism contributes modestly to adaptation and chromatic and spatiotemporal processing (for review, see Burkhardt, 1993). Although it has been shown that squirrel cones have surrounds (Leeper and Charlton, 1985), very few physiological recordings have been made in cat or monkey cones (Nelson, 1977; Schnapf et al., 1990; Schneeweis and Schnapf, 1999), and it is not known if their receptive fields have surrounds.

Cone bipolar cells in nonmammalian retina have an antagonistic surround probably generated by horizontal cells, amacrine cells, and horizontal cell feedback to cones (Wu, 1994). Antagonistic surround responses have been measured in cat bipolar cells (Nelson et al., 1981), but only very rarely. Preliminary intracellular recordings from several primate diff use bipolar cells and a single midget bipolar cell also reveal strong antagonistic surrounds (Packer et al., 1999; Dacey and Lee, 1999). The surrounds of bipolar cells are therefore a plausible source of nearest neighbor contribution that we infer from our measurements.

\section{Small or irregular surrounds generated by amacrine cells}

Amacrine cells frequently make reciprocal synapses with midget bipolar cells in the fovea, and this feedback pathway is very spatially localized (Calkins and Sterling, 1996). Electron microscopy of serial sections has shown that the number of amacrine synapses on to human parafoveal midget ganglion cells is roughly equal to the number of bipolar ribbon inputs (Kolb and Dekorver, 1991). Based on these anatomical findings, spatially localized amacrine-bipolar cell feedback pathways and inhibitory connections between narrow field amacrine cells and midget ganglion cells must also be considered as possible sources of nearest neighbor contribution.

\section{Relationship between physiology and visual performance}

The most sensitive indicator of neural spatial filtering at high frequencies is the psychophysical measurement of contrast sensitivity with interference fringes. Experiments of this kind have shown a steep attenuation of spatial frequencies at $\sim 40-60 \mathrm{c} /{ }^{\circ}$ (Campbell and Green, 1965; Williams, 1985b; Sekiguchi et al., 1993; He and MacLeod, 1996). Spatial frequency discrimination experiments demonstrating good discrimination up to the resolution limit, together with the results of contrast and spatial frequency matching after very high spatial frequency adaptation, also suggest that the smallest cortical receptive fields used in human spatial vision have a cutoff near $60 \mathrm{c} /{ }^{\circ}$ (Smallman et al., 1996). Our results show that parvocellular LGN neurons respond reliably to much higher spatial frequencies (up to and beyond 100 $\mathrm{c} /{ }^{\circ}$ ). This suggests that high spatial frequency information in the range of the human resolution limit conveyed to the cortex by LGN cells does not result in a correspondingly fine subjective experience of spatial patterns.

However, Williams (1985a) showed that observers in a forced choice experiment could detect interference fringes at spatial frequencies at least as high as $150 \mathrm{c} /{ }^{\circ}$, well above the Nyquist limit set by the array of photoreceptors at about $60 \mathrm{c} /{ }^{\circ}$ in humans. The high-frequency responses reported here in primates reveal the signals available at the level of the LGN for that psychophysical detection performance. At spatial frequencies exceeding the cone Nyquist limit, human observers see an irregular pattern of stripes lower in spatial frequency than the stimulus caused by aliasing. We have shown here that LGN neurons can convey information about such high-frequency fringes, but the cortex lacks the machinery to reconstruct such undersampled fringes correctly from the array of LGN neurons. Consequently, these fringes can be detected, but their patterns are not resolved.

\section{REFERENCES}

Attwell D (1986) The Sharpey-Schafer lecture. Ion channels and signal processing in the outer retina. Q J Exp Physiol 71:497-536.

Baldridge WH, Vaney DI, Weiler R (1998) The modulation of intercellular coupling in the retina. Semin Cell Dev Biol 9:311-318.

Baylor DA, Nunn BJ, Schnapf JL (1987) Spectral sensitivity of cones of the monkey Macaca fascicularis. J Physiol (Lond) 390:145-160.

Bracewell RN (1986) The Fourier transform and its applications. New York: McGraw-Hill.

Burkhardt DA (1993) Synaptic feedback, depolarization, and color opponency in cone photoreceptors. Vis Neurosci 10:981-989.

Calkins DJ, Sterling P (1996) Absence of spectrally specific lateral inputs to midget ganglion cells in primate retina. Nature 381:613-615.

Calkins DJ, Schein SJ, Tsukamoto Y, Sterling P (1994) M and L cones in macaque fovea connect to midget ganglion cells by different numbers of excitatory synapses. Nature 371:70-72.

Campbell FW, Green DG (1965) Optical and retinal factors affecting visual resolution. J Physiol (Lond) 181:576-593.

Campbell FW, Gubisch RW (1966) Optical quality of the human eye. J Physiol (Lond) 186:558-578.

Chen B, Makous W, Williams DR (1993) Serial spatial filters in vision. Vision Res 33:413-427.

Cohen AI (1965) Some electron microscopic observations on interreceptor contacts in the human and macaque retinae. $J$ Anat 99:595-610.

Croner LJ, Kaplan E (1995) Receptive fields of P and M ganglion cells across the primate retina. Vision Res 35:7-24.

Dacey DM, Brace S (1992) A coupled network for parasol but not midget ganglion cells in the primate retina. Vis Neurosci 9:279-90.

Dacey DM, Lee BB (1999) Functional architecture of cone signal pathways in the primate retina. In: Color vision: from genes to perception (Gegenfurtner KR, Sharpe LT, eds), pp 181-202. New York: Cambridge UP.

Dacey DM, Lee BB, Stafford DK, Pokorny J, Smith VC (1996) Horizontal cells of the primate retina: cone specificity without spectral opponency. Science 271:656-659.

Derrington AM, Lennie P (1984) Spatial and temporal contrast sensitivities of neurones in lateral geniculate nucleus of macaque. J Physiol (Lond) 357:219-240.

Enoch JM, Tobey Jr FL (1981) Vertebrate photoreceptor optics. Berlin: Springer.

Enroth-Cugell C, Robson JG (1966) The contrast sensitivity of retinal ganglion cells of the cat. J Physiol (Lond) 187:517-552.

He S, MacLeod DI (1996) Local luminance nonlinearity and receptor aliasing in the detection of high-frequency gratings. J Opt Soc Am A 13:1139-1151.

Hirsch J, Miller WH (1987) Does cone positional disorder limit resolution? J Opt Soc Am A 4:1481-1492.

Hsu AC-C (1998) Optimal transfer of form and color information through the primate retina. PhD thesis, University of Pennsylvania. 
Kaplan E, Shapley RM (1982) X and Y cells in the lateral geniculate nucleus of macaque monkeys. J Physiol (Lond) 330:125-143.

Kolb H, Dekorver L (1991) Midget ganglion cells of the parafovea of the human retina: a study by electron microscopy and serial section reconstructions. J Comp Neurol 303:617-636.

Leeper HF, Charlton JS (1985) Response properties of horizontal cells and photoreceptor cells in the retina of the tree squirrel, Sciurus carolinensis. J Neurophysiol 54:1157-1166.

Liang J, Williams DR, Miller DT (1997) Supernormal vision and highresolution retinal imaging through adaptive optics. J Opt Soc Am A 14:2884-2892.

MacLeod DI, Williams DR, Makous W (1992) A visual nonlinearity fed by single cones. Vision Res 32:347-363.

McMahon MJ, Lankheet MJM, Lennie P, Williams DR (1995) Fine structure of P-cell receptive fields in the fovea revealed by laser interferometry. Invest Ophthalmol Vis Sci [Suppl] 36/4:S4.

Nelson R (1977) Cat cones have rod input: a comparison of the response properties of cones and horizontal cell bodies in the retina of the cat. J Comp Neurol 172:109-135.

Nelson R, Kolb H, Robinson MM, Mariani AP (1981) Neural circuitry of the cat retina: cone pathways to ganglion cells. Vision Res 21:1527-1536.

National Institutes of Health. (1994) Preparation and maintenance of higher mammals during neuroscience experiments (Report of a NIH Workshop No. 94-3207).

Packer O, Hendrickson AE, Curcio CA (1989) Photoreceptor topography of the retina in the adult pigtail macaque (Macaca nemestrina). J Comp Neurol 288:165-183.

Packer OS, Diller L, Lee BB, Dacey DM (1999) Diffuse cone bipolar cells in macaque monkey retina are spatially opponent. Invest Ophthalmol Vis Sci [Suppl] 40/4:S790.

Pask C, Stacey A (1998) Optical properties of retinal photoreceptors and the Campbell effect. Vision Res 38:953-961.

Raviola E, Gilula NB (1973) Gap junctions between photoreceptor cells in the vertebrate retina. Proc Natl Acad Sci USA 70:1677-1681.

Schnapf JL, Nunn BJ, Meister M, Baylor DA (1990) Visual transduction in cones of the monkey Macaca fascicularis. J Physiol (Lond) 427:681-713.

Schneeweis DM, Schnapf JL (1999) The photovoltage of macaque cone photoreceptors: adaptation, noise, and kinetics. J Neurosci 19:1203-1216.

Sekiguchi N, Williams DR, Brainard DH (1993) Efficiency in detection of isoluminant and isochromatic interference fringes. J Opt Soc Am A 10:2118-2133.

Shapley R, Kaplan E, Soodak R (1981) Spatial summation and contrast sensitivity of $\mathrm{X}$ and $\mathrm{Y}$ cells in the lateral geniculate nucleus of the macaque. Nature 292:543-545.

Smallman HS, MacLeod DI, He S, Kentridge RW (1996) Fine grain of the neural representation of human spatial vision. J Neurosci $16: 1852-1859$.

Soodak RE (1986) Two-dimensional modeling of visual receptive fields using Gaussian subunits. Proc Natl Acad Sci USA 83:9259-9263.

Soodak RE, Shapley RM, Kaplan E (1987) Linear mechanism of orientation tuning in the retina and lateral geniculate nucleus of the cat. J Neurophysiol 58:267-275.

Soodak RE, Shapley RM, Kaplan E (1991) Fine structure of receptivefield centers of X and Y cells of the cat. Vis Neurosci 6:621-628.

Stockman A, MacLeod DI, Johnson NE (1993) Spectral sensitivities of the human cones. J Opt Soc Am A 10:2491-2521.

Tessier-Lavigne M, Attwell D (1988) The effect of photoreceptor coupling and synapse nonlinearity on signal:noise ratio in early visual processing. Proc R Soc Lond B Biol Sci 234:171-197.

Thibos LN, Levick WR (1983) Bimodal receptive fields of cat retinal ganglion cells. Vision Res 23:1561-1572.

Tsukamoto Y, Masarachia P, Schein SJ, Sterling P (1992) Gap junctions between the pedicles of macaque foveal cones. Vision Res 32:1809-1815.

Vaney DI (1996) Cell coupling in the retina. In: Gap junctions in the nervous system (Spray DC, Dermietzel R, eds), pp 79-102. Georgetown, TX: R. G. Landes.

Walraven J, Enroth-Cugell C, Hood DC, MacLeod DIA, Schnapf JL (1990) The control of visual sensitivity: receptoral and postreceptoral processes. In: Visual perception: the neurophysiological foundations (Spillmann L, Werner JS, eds), pp 53-101. San Diego: Academic.

Wässle H, Grünert U, Martin PR, Boycott BB (1994) Immunocytochemical characterization and spatial distribution of midget bipolar cells in the macaque monkey retina. Vision Res 34:561-579.

Wässle H, Grünert U, Röhrenbeck J, Boycott BB (1989) Cortical magnification factor and the ganglion cell density of the primate retina. Nature 341:643-646.

Williams DR (1985a) Aliasing in human foveal vision. Vision Res 25:195-205.

Williams DR (1985b) Visibility of interference fringes near the resolution limit. J Opt Soc Am A 2:1087-1093.

Williams DR, Sekiguchi N, Haake W, Brainard DH, Packer O (1991) The cost of trichromacy for spatial vision. In: From pigments to perception: advances in understanding visual processes (Valberg A, Lee BB, eds), pp 11-22. New York: Plenum.

Williams DR, Brainard DH, McMahon MJ, Navarro R (1994) Doublepass and interferometric measures of the optical quality of the eye. $J$ Opt Soc Am A 11:3123-3135.

Wu SM (1994) Synaptic transmission in the outer retina. Annu Rev Physiol 56:141-168.

Yellott Jr JI (1983) Spectral consequences of photoreceptor sampling in the rhesus retina. Science 221:382-385. 